Local property

\title{
On the local property for positivity preserving coercive forms
}

\author{
Byron Schmuland
}

\begin{abstract}
We show that, under mild conditions, two well-known definitions for the local property of a Dirichlet form are equivalent. We also show that forms that come from differential operators are local.
\end{abstract}

1991 AMS Subject Classification: 31C25

The purpose of this paper is to clarify the relationship between two different notions of locality that have appeared in the literature of Dirichlet forms. The first is a slightly modified version of the definition of locality found in the book of Bouleau and Hirsch $[\mathrm{BH}$ 91; Chapter I, Corollary 5.1.4], while the second comes from the book of Ma and Röckner [MR 92; Chapter V, Proposition 1.2]. But here we do not assume that the form satisfies any normal contraction property, but only that it is positivity preserving (see Definition 0.1 below).

Let $(E, \mathcal{F}, m)$ be a measure space, and suppose $(\mathcal{E}, D(\mathcal{E}))$ is a densely defined, closed, bilinear form on $L^{2}(E, \mathcal{F}, m)$. Following [MR 92], we call such a form $(\mathcal{E}, D(\mathcal{E}))$ coercive if $\mathcal{E}(u, u) \geq 0$ for all $u \in D(\mathcal{E})$, and for some $K \geq 1$ we have,

$$
|\mathcal{E}(u, v)| \leq K \mathcal{E}_{1}(u, u)^{1 / 2} \mathcal{E}_{1}(v, v)^{1 / 2} \text { for all } u, v \in D(\mathcal{E}),
$$

where we define $\mathcal{E}_{1}(u, v):=\mathcal{E}(u, v)+(u, v)_{L^{2}(E ; m)}$.

Definition 0.1. The following are various contraction properties that the form $(\mathcal{E}, D(\mathcal{E}))$ may satisfy:

(C1) If $u \in D(\mathcal{E})$, then $u^{+} \in D(\mathcal{E})$ and $\mathcal{E}\left(u^{+}, u^{-}\right) \leq 0$.

(C2) If $u \in D(\mathcal{E})$, then $u \wedge 1 \in D(\mathcal{E})$ and $\mathcal{E}(u \wedge 1, u-u \wedge 1) \geq 0$.

(C3) If $u \in D(\mathcal{E})$, then $u \wedge 1 \in D(\mathcal{E})$ and $\mathcal{E}(u-u \wedge 1, u \wedge 1) \geq 0$.

Condition (C1) is equivalent with the positivity of the associated semigroup $\left(T_{t}\right)_{t \geq 0}$, (and hence also the dual semigroup $\left(\hat{T}_{t}\right)_{t \geq 0}$ ); that is, for $u \in L^{2}$ if $0 \leq u$, then $0 \leq T_{t} u$. Hence, a form $(\mathcal{E}, D(\mathcal{E})$ ) satisfying $(\mathrm{C} 1)$ is called positivity preserving. Condition (C2) is equivalent to the Markov property for $\left(T_{t}\right)_{t \geq 0}$; that is, for $u \in L^{2}$ if $0 \leq u \leq 1$, then $0 \leq T_{t} u \leq 1$, while condition (C3) is equivalent to the Markov property for the dual semigroup $\left(\hat{T}_{t}\right)_{t \geq 0}$. We note that either of $(\mathrm{C} 2)$ or $(\mathrm{C} 3)$ implies $(\mathrm{C} 1)$. A form $(\mathcal{E}, D(\mathcal{E}))$ 
satisfying either of $(\mathrm{C} 2)$ or $(\mathrm{C} 3)$ is called a semi-Dirichlet form, while if it satisfies both (C2) and (C3) it is called a Dirichlet form. Contraction properties and their relation with associated semigroups are studied, for example, in [F 80], [BH 91; Chapter I.3], [MR 92; Chapter I.4]. These references also discuss Markov processes associated to Dirichlet forms. A treatment of associated Markov processes for semi-Dirichlet forms can be found in [MOR 93], and the positivity preserving case is covered in [MR 93]. From now on $(\mathcal{E}, D(\mathcal{E})$ ) will always denote a coercive form and we always equip $D(\mathcal{E})$ with the norm coming from the inner product $\mathcal{E}_{1}$. Ancona has shown $[$ An 76$]$ that if $(\mathcal{E}, D(\mathcal{E}))$ is a positivity preserving coercive form, then the mapping $u \rightarrow u^{+}$is strongly continuous on $D(\mathcal{E})$. We shall use this result throughout.

Notation. For functions $u, v$ on $E$, notation like $u \leq v$ or $u=v$ is always to be understood in the $m$-almost everywhere sense. We will often omit the qualifier " $m$-a.e.".

\section{The two local properties}

In this section we compare two definitions of locality for coercive forms (see Definition 1.2 below). One of them, (L2), makes topological assumptions on the space $E$, while the other, (L1), has the advantage of making sense on any measurable space $E$. Nevertheless, in practice, it is the relationship between the local property and the topology on $E$ that is of interest. In particular, if the topological assumption $(\mathrm{T})$ holds and if $(\mathcal{E}, D(\mathcal{E}))$ is a quasi-regular semi-Dirichlet form, then property (L2) is equivalent to the continuity in $E$ of the sample paths of the associated Markov process (cf. [MR 92; Chapter V, Theorem 1.5] and [MOR 93; Remark 3.10]). Hence this is also true for quasi-regular positivity preserving coercive forms and their associated $h$-processes [MR 93].

Definition 1.1. We say that condition (T) holds if $E$ is a topological Hausdorff space, $m$ is a $\sigma$-finite Borel measure on $E$, and $(\mathcal{E}, D(\mathcal{E}))$ is a coercive form on $L^{2}(E, \mathcal{B}(E), m)$.

When (T) holds, for any $m$-square integrable Borel function $u$ we define the support of $u, \operatorname{supp}[u]$, by

$$
\operatorname{supp}[u]:=\left(\cup_{\alpha \in \Gamma} \mathcal{O}_{\alpha}\right)^{c},
$$

where the union is over all open sets $\mathcal{O}_{\alpha}$ such that $\int_{\mathcal{O}_{\alpha}} u^{2} d m=0$. We note that if $u=v$ $m$-a.e., then $\operatorname{supp}[u]=\operatorname{supp}[v]$, so the support is well-defined on $L^{2}(E, \mathcal{B}(E), m)$.

Definition 1.2. Here are the two defintions of locality that we wish to compare.

(L1) The form $(\mathcal{E}, D(\mathcal{E}))$ has local property (L1) if, for $u, v \in D(\mathcal{E})$ with $u v=0$, we have $\mathcal{E}(u, v)=0$.

(L2) Suppose that (T) holds. The form $(\mathcal{E}, D(\mathcal{E}))$ has local property (L2) if, for $u, v \in D(\mathcal{E})$ with $\operatorname{supp}[u] \cap \operatorname{supp}[v]=\emptyset$, we have $\mathcal{E}(u, v)=0$. 
Definition 1.3. Suppose that (T) holds. Then we define the following:

(a) For any closed set $F \subseteq E$ we define

$$
D(\mathcal{E})_{F}:=\left\{u \in D(\mathcal{E}) \mid u=0 m \text { - a.e. on } F^{c}\right\} .
$$

(b) An increasing sequence of closed sets $\left(F_{k}\right)_{k \in \mathbb{N}}$ is called an $\mathcal{E}$-nest if $\cup_{k} D(\mathcal{E})_{F_{k}}$ is dense in $D(\mathcal{E})$.

(c) A function $f: E \rightarrow \mathbb{R}$ is called $\mathcal{E}$-quasi-continuous if there exists an $\mathcal{E}$-nest $\left(F_{k}\right)_{k \in \mathbb{N}}$ such that $\left.f\right|_{F_{k}}$ is continuous for each $k \in \mathbb{N}$.

Proposition 1.4. Suppose that $(\mathcal{E}, D(\mathcal{E}))$ is a positivity preserving coercive form and that assumption (T) holds. If $E$ is strongly Lindelöf, then (L1) $\Rightarrow$ (L2). On the other hand, if the following two conditions hold, then (L2) $\Rightarrow$ (L1).

(a) Every $u \in D(\mathcal{E})$ has an $\mathcal{E}$-quasi-continuous $m$-version $\tilde{u}$.

(b) There exists an $\mathcal{E}$-quasi-continuous m-version $\tilde{h}$ of some $h \in D(\mathcal{E})$, and an $\mathcal{E}$-nest $\left(F_{k}\right)_{k \in \mathbb{N}}$ so that $\left.\tilde{h}\right|_{F_{k}}$ is continuous and $\inf _{z \in F_{k}} \tilde{h}(z):=\delta_{k}>0$ for each $k \in \mathbb{N}$.

\section{Comment.}

(i) For a semi-Dirichlet form $(\mathcal{E}, D(\mathcal{E})$ ), conditions (a) and (b) above follow if there is a dense set $D$ in $D(\mathcal{E})$ such that every $u \in D$ has an $\mathcal{E}$-quasi-continuous $m$-version $\tilde{u}$.

(ii) By [MR 93], conditions (a) and (b) are true for any quasi-regular positivity preserving coercive form.

Proof. If $E$ is strongly Lindelöf, the open set $\cup_{\alpha \in \Gamma} \mathcal{O}_{\alpha}$ has a countable subcover $\left(\mathcal{O}_{n}\right)_{n \in \mathbb{N}}$, and so

$$
\int_{\cup_{\alpha \in \Gamma} \mathcal{O}_{\alpha}} u^{2} d m=\int_{\cup_{n \in \mathbb{N}} \mathcal{O}_{n}} u^{2} d m \leq \sum_{n \in \mathbb{N}} \int_{\mathcal{O}_{n}} u^{2} d m=0 .
$$

That is, $u=0 \mathrm{~m}$-a.e. on the complement of its support. Therefore, we have $u v=0$ whenever $\operatorname{supp}[u] \cap \operatorname{supp}[v]=\emptyset$, and so the implication (L1) $\Rightarrow$ (L2) is trivial.

Now suppose that (L2), as well as conditions (a) and (b), hold and let $u, v \in D(\mathcal{E})$ with $u v=0$. We must show that $\mathcal{E}(u, v)=0$, and by separate consideration of $\mathcal{E}\left(u^{+}, v^{-}\right)$, $\mathcal{E}\left(u^{+}, v^{+}\right), \mathcal{E}\left(u^{-}, v^{+}\right)$, and $\mathcal{E}\left(u^{-}, v^{-}\right)$, we may assume that $u \geq 0$ and $v \geq 0$.

Let $\left(F_{k}\right)_{k \in \mathbb{N}}$ be an $\mathcal{E}$-nest and $\tilde{u}$ an $\mathcal{E}$-quasi-continuous $m$-version of $u$ so that $\left.\tilde{u}\right|_{F_{k}}$ is continuous for each $k \in \mathbb{N}$. By intersecting with the nest in (b), we may also assume that $\left.\tilde{h}\right|_{F_{k}}$ is continuous and $\inf _{z \in F_{k}} \tilde{h}(z):=\delta_{k}>0$ for each $k \in \mathbb{N}$. Since $\left(F_{k}\right)_{k \in \mathbb{N}}$ is an $\mathcal{E}$-nest, we can find sequences $\left(u_{n}\right)_{n \in \mathbb{N}}$ and $\left(v_{m}\right)_{m \in \mathbb{N}}$ in $\cup_{k} D(\mathcal{E})_{F_{k}}$ so that $u_{n} \rightarrow u$ and $v_{m} \rightarrow v$ in $D(\mathcal{E})$. Since the mapping $u \rightarrow u^{+}$is strongly continuous, we have $u_{n}^{+} \rightarrow u^{+}=u$ and $v_{m}^{+} \rightarrow v^{+}=v$. It also holds that $u_{n}^{+} \wedge u \rightarrow u$ and $v_{m}^{+} \wedge v \rightarrow v$ in $D(\mathcal{E})$. So without loss of generality we may assume that $0 \leq u_{n} \leq u$ and $0 \leq v_{m} \leq v$ for all $n, m \in \mathbb{N}$. 
Fix $n, m \in \mathbb{N}$ and choose $k \in \mathbb{N}$ so large that $u_{n}, v_{m} \in D(\mathcal{E})_{F_{k}}$. Let $z \in \operatorname{supp}\left[\left(u_{n}-\right.\right.$ $\varepsilon h)^{+}$. Then for every open neighborhood $\mathcal{O}$ of $z$ we have

$$
\int_{\mathcal{O}}\left(\left(u_{n}-\varepsilon h\right)^{+}\right)^{2} d m>0
$$

which implies

$$
\int_{\mathcal{O} \cap F_{k}}\left((u-\varepsilon h)^{+}\right)^{2} d m>0
$$

This means that the set

$$
\mathcal{O} \cap F_{k} \cap\{z \mid \tilde{u}(z)-\varepsilon \tilde{h}(z)>0\} \subseteq \mathcal{O} \cap F_{k} \cap\left\{z \mid \tilde{u}(z) \geq \varepsilon \delta_{k}\right\}
$$

is non-empty. Since this holds for every neighborhood $\mathcal{O}$, it follows that $z$ belongs to the closed set $F_{k} \cap\left\{z \mid \tilde{u}(z) \geq \varepsilon \delta_{k}\right\}$, that is,

$$
\operatorname{supp}\left[\left(u_{n}-\varepsilon h\right)^{+}\right] \subseteq F_{k} \cap\left\{\tilde{u} \geq \varepsilon \delta_{k}\right\}
$$

On the other hand, $u v_{m}=0$ so

$$
0=\int_{\left\{\tilde{u}>\varepsilon \delta_{k} / 2\right\}} u v_{m} d m \geq\left(\varepsilon \delta_{k} / 2\right) \int_{\left\{\tilde{u}>\varepsilon \delta_{k} / 2\right\}} v_{m} d m
$$

which gives

$$
\int_{\left\{\tilde{u}>\varepsilon \delta_{k} / 2\right\}} v_{m}^{2} d m=0
$$

Since we already know that $\int_{F_{k}^{c}} v_{m}^{2} d m=0$, we have

$$
\int_{\left\{\tilde{u}>\varepsilon \delta_{k} / 2\right\} \cup F_{k}^{c}} v_{m}^{2} d m=0,
$$

and, as the set $\left\{\tilde{u}>\varepsilon \delta_{k} / 2\right\} \cup F_{k}^{c}$ is open, we conclude that $\operatorname{supp}\left[v_{m}\right] \subseteq\left\{\tilde{u} \leq \varepsilon \delta_{k} / 2\right\} \cap F_{k}$. In other words, $\operatorname{supp}\left[v_{m}\right] \cap \operatorname{supp}\left[\left(u_{n}-\varepsilon h\right)^{+}\right]=\emptyset$, so by (L2)

$$
\mathcal{E}\left(\left(u_{n}-\varepsilon h\right)^{+}, v_{m}\right)=0 .
$$

Letting $\varepsilon \rightarrow 0$, then $n \rightarrow \infty$, and finally $m \rightarrow \infty$ gives the result $\mathcal{E}(u, v)=0$.

\section{Examples of local forms}

This section is dedicated to showing that forms given by differential operators are local in the (L1) sense. This result is not surprising, but it is, nevertheless, not trivial to check since it involves the behaviour of the form $\mathcal{E}$, not only for functions $u$ in a nice core, but also for their positive parts $u^{+}$.

The following example is the prototype for a non-symmetric form on a (possibly) infinite dimensional space. This example suggested the form of Proposition 2.3 which follows. 
Example 2.1. (cf. [MR 92; Chapter II, Section 3])

Let $E$ be a locally convex topological vector space which, in addition, is Souslin. Let $m$ be a finite positive measure on $\mathcal{B}(E)$ such that $\operatorname{supp}[m]=E$. Let $E^{\prime}$ denote the dual of $E$ and $E^{\prime}\langle,\rangle_{E}: E^{\prime} \times E \rightarrow \mathbb{R}$ the corresponding dualization. Define a linear space of functions on $E$ by

$$
\mathcal{F} C_{b}^{\infty}:=\left\{f\left(l_{1}, \ldots, l_{m}\right) \mid m \in \mathbb{N}, f \in C_{b}^{\infty}\left(\mathbb{R}^{m}\right), l_{1}, \ldots, l_{m} \in E^{\prime}\right\} .
$$

Here $C_{b}^{\infty}\left(\mathbb{R}^{m}\right)$ denotes the set of all infinitely differentiable functions on $\mathbb{R}^{m}$ with all partial derivatives bounded. By the Hahn-Banach theorem, $\mathcal{F} C_{b}^{\infty}$ separates points of $E$; hence, since $\mathcal{B}(E)=\sigma\left(E^{\prime}\right)$, the space $\mathcal{F} C_{b}^{\infty}$ is dense in $L^{2}(E ; m)$ by the monotone class theorem. Define for $u \in \mathcal{F} C_{b}^{\infty}$ and $k \in E$,

$$
\frac{\partial u}{\partial k}(z):=\left.\frac{d}{d s} u(z+s k)\right|_{s=0}, z \in E .
$$

Observe that if $u=f\left(l_{1}, \ldots, l_{m}\right)$, then

$$
\frac{\partial u}{\partial k}=\sum_{i=1}^{m} \frac{\partial f}{\partial x_{i}}\left(l_{1}, \ldots, l_{m}\right)_{E^{\prime}}\left\langle l_{i}, k\right\rangle_{E} \in \mathcal{F} C_{b}^{\infty} .
$$

Now assume that there exists a separable real Hilbert space $\left(H,\langle,\rangle_{H}\right)$ densely and continuously embedded into $E$. Identifying $H$ with its dual gives $E^{\prime} \subset H \subset E$, so that $E^{\prime}\langle,\rangle_{E}$ restricted to $E^{\prime} \times H$ coincides with $\langle,\rangle_{H}$. By $(2.3)$, for each $u \in \mathcal{F} C_{b}^{\infty}$ and $z \in E$, the map $h \rightarrow(\partial u / \partial h)(z)$ is a continuous linear functional on $H$. Define $\nabla u(z)$ by

$$
\langle\nabla u(z), h\rangle_{H}=\frac{\partial u}{\partial h}(z), h \in H .
$$

Now suppose we are given $A \in L^{\infty}(E \rightarrow \mathcal{L}(H) ; \mu), b, d \in L^{\infty}(E \rightarrow H ; \mu)$, and $c \in$ $L^{\infty}(E ; \mu)$. Define for $u, v \in \mathcal{F} C_{b}^{\infty}$,

$$
\begin{aligned}
\mathcal{E}(u, v) & =\int\langle A \nabla u, \nabla v\rangle_{H} d m+\int\langle b, \nabla u\rangle_{H} v d m \\
& +\int u\langle d, \nabla v\rangle_{H} d m+\int u v c d m .
\end{aligned}
$$

We also suppose that the form $\left(\mathcal{E}, \mathcal{F} C_{b}^{\infty}\right)$ is positive definite and closable in $L^{2}(E ; m)$, and that for $u, v \in \mathcal{F} C_{b}^{\infty}$ the following hold:

(a) $\langle A \nabla u, \nabla u\rangle_{H} \geq 0$ and $\left|\langle A \nabla u, \nabla v\rangle_{H}\right| \leq K\langle A \nabla u, \nabla u\rangle_{H}^{1 / 2}\langle A \nabla v, \nabla v\rangle_{H}^{1 / 2}$, for some $K \geq 1$.

(b) $\int\left(\left|\langle b, \nabla u\rangle_{H} v\right|+\left|u\langle d, \nabla v\rangle_{H}\right|+|u v c|\right) d m \leq K \mathcal{E}_{1}(u, u)^{1 / 2} \mathcal{E}_{1}(v, v)^{1 / 2}$, for some $K \geq 1$.

If these conditions hold, then the closure $(\mathcal{E}, D(\mathcal{E}))$ is coercive, and Proposition 2.3 below shows that $(\mathcal{E}, D(\mathcal{E}))$ is local. Indeed, assumptions (a) and (b) above imply that conditions (a) and (b) in the statement of Proposition 2.3 hold, while conditions (c)-(f) follow easily from pointwise multiplication and the chain rule on $\mathcal{F} C_{b}^{\infty}$. Therefore Proposition 2.3 is 
valid for $(\mathcal{E}, D(\mathcal{E}))$ and it follows that $(\mathcal{E}, D(\mathcal{E}))$ satisfies the contraction property $(\mathrm{C} 1)$, and also the local property (L1).

Lemma 2.2. Suppose $(\mathcal{E}, D(\mathcal{E}))$ is a closed, coercive form on $L^{2}(E ; m)$. If there is a dense set $D$ in $D(\mathcal{E})$ such that $u \in D$ implies $u^{+} \in D(\mathcal{E})$ and $\mathcal{E}\left(u^{+}, u^{-}\right)=0$, then $(\mathcal{E}, D(\mathcal{E}))$ is positivity preserving and satisfies the local property (L1).

Proof. For $u \in D$ we have $u^{+} \in D(\mathcal{E})$ and $\mathcal{E}\left(u^{+}, u^{-}\right) \leq 0$. It is not hard to show (cf. [MR 92; Chapter I, Lemma 4.9]) that this carries over to all of $D(\mathcal{E})$, that is, $(\mathcal{E}, D(\mathcal{E}))$ is positivity preserving. By hypothesis and Ancona's continuity result we have, in fact, $\mathcal{E}\left(u^{+}, u^{-}\right)=0$ for all $u \in D(\mathcal{E})$. Now let $u, v \in D(\mathcal{E})$ so that $u v=0$. We must show that $\mathcal{E}(u, v)=0$, and by separate consideration of $\mathcal{E}\left(u^{+}, v^{-}\right), \mathcal{E}\left(u^{+}, v^{+}\right), \mathcal{E}\left(u^{-}, v^{+}\right)$, and $\mathcal{E}\left(u^{-}, v^{-}\right)$, we may assume that $u \geq 0$ and $v \geq 0$. Then $u=(u-v)^{+}$and $v=(u-v)^{-}$so $\mathcal{E}(u, v)=0$.

Proposition 2.3. Suppose $(\mathcal{E}, D(\mathcal{E}))$ is a closed, coercive form on $L^{2}(E ; m)$. Also, suppose that $D$ is a dense, linear subspace of $D(\mathcal{E})$, consisting of bounded functions and that $D$ is closed under pointwise multiplication. Suppose also that for $u, v \in D$ we have $\mathcal{E}(u, v)=\int \sum_{i=1}^{4} \Gamma_{i}(u, v) d m$, where $\left(\Gamma_{i}\right)_{i=1}^{4}$ are bilinear maps from $D \times D$ into $L^{1}(E ; m)$ satisfying for all $u, v, w \in D$ :

(a) $\Gamma_{1}(u, u) \geq 0$ and $\left|\Gamma_{1}(u, v)\right| \leq K \Gamma_{1}(u, u)^{1 / 2} \Gamma_{1}(v, v)^{1 / 2}$, for some $K \geq 1$.

(b) $\int\left(\left|\Gamma_{2}(u, v)\right|+\left|\Gamma_{3}(u, v)\right|+\left|\Gamma_{4}(u, v)\right|\right) d m \leq K \mathcal{E}_{1}(u, u)^{1 / 2} \mathcal{E}_{1}(v, v)^{1 / 2}$, for some $K \geq 1$.

(c) $\Gamma_{1}(u v, w)=u \Gamma_{1}(v, w)+v \Gamma_{1}(u, w)$.

(d) $\Gamma_{2}(u v, w)=u \Gamma_{2}(v, w)+v \Gamma_{2}(u, w)$, and $\Gamma_{2}(w, u v)=u \Gamma_{2}(w, v)=v \Gamma_{2}(w, u)$.

(e) $\Gamma_{3}(u v, w)=u \Gamma_{3}(v, w)=v \Gamma_{3}(u, w)$, and $\Gamma_{3}(w, u v)=u \Gamma_{3}(w, v)+v \Gamma_{3}(w, u)$.

(f) $\Gamma_{4}(u v, w)=u \Gamma_{4}(v, w)=v \Gamma_{4}(u, w)$, and $\Gamma_{4}(w, u v)=u \Gamma_{4}(w, v)=v \Gamma_{4}(w, u)$.

Then $(\mathcal{E}, D(\mathcal{E}))$ is positivity preserving and satisfies the local property (L1).

Proof. We begin by noting that conditions (a) and (b) imply that each of the bilinear maps $\left(\Gamma_{i}\right)_{i=1}^{4}$ can be extended continuously to all of $D(\mathcal{E})$ in such a way that the representation $\mathcal{E}(u, v)=\int \sum_{i=1}^{4} \Gamma_{i}(u, v) d m$ holds on all of $D(\mathcal{E})$.

Suppose that $P$ is a polynomial on $\mathbb{R}$ with $P(0)=0$. Then $P(x) / x$ is a polynomial, which, by the mean value theorem, satisfies

$$
|P(x) / x| \leq \sup _{0 \leq|\xi| \leq|x|}\left|P^{\prime}(\xi)\right| .
$$

For $u, w \in D$ and such a polynomial $P$, we have $P(u) \in D(\mathcal{E})$ and, by conditions (c)-(f),

$$
\left|\Gamma_{1}(P(u), w)+\Gamma_{2}(P(u), w)\right|=\left|P^{\prime}(u)\left(\Gamma_{1}(u, w)+\Gamma_{2}(u, w)\right)\right|,
$$

and

$$
\left|\Gamma_{3}(P(u), w)+\Gamma_{4}(P(u), w)\right|=\left|(P(u) / u)\left(\Gamma_{3}(u, w)+\Gamma_{4}(u, w)\right)\right| .
$$


From (2.6)-(2.8) and condition (b), there exists a constant $c>0$ such that

$$
|\mathcal{E}(P(u), w)| \leq c \sup _{0 \leq|\xi| \leq\|u\|_{\infty}}\left|P^{\prime}(\xi)\right| \mathcal{E}_{1}(u, u)^{1 / 2} \mathcal{E}_{1}(w, w)^{1 / 2} .
$$

Adding $\int P(u) w d m$, and using (2.9) gives the bound

$$
\left|\mathcal{E}_{1}(P(u), w)\right| \leq\left(c \sup _{0 \leq|\xi| \leq\|u\|_{\infty}}\left|P^{\prime}(\xi)\right|+1\right) \mathcal{E}_{1}(u, u)^{1 / 2} \mathcal{E}_{1}(w, w)^{1 / 2} .
$$

Taking the supremum over $w \in D$ with $\mathcal{E}_{1}(w, w)^{1 / 2} \leq 1$, yields

$$
\mathcal{E}_{1}(P(u), P(u))^{1 / 2} \leq\left(c \sup _{0 \leq|\xi| \leq\|u\|_{\infty}}\left|P^{\prime}(\xi)\right|+1\right) \mathcal{E}_{1}(u, u)^{1 / 2} .
$$

Fix $u \in D$ and set $I:=\left[-\|u\|_{\infty},\|u\|_{\infty}\right]$. Define $f_{n}$ to be the function on $\mathbb{R}$ with $f_{n}(x)=1$ for $x \leq-1 / n, f_{n}(x)=0$ for $x \geq 0$, and so that $f_{n}$ is linear between $-1 / n$ and 0 . By the Stone-Weierstrass theorem we can find a polynomial $Q_{n}^{\prime}$ so that $\left|Q_{n}^{\prime}(x)-f_{n}(x)\right| \leq 1 / n$ for all $x \in I$. We set $P_{n}^{\prime}=1-Q_{n}^{\prime}$, and define two sequences of polynomials that vanish at zero by setting

$$
Q_{n}(x):=\int_{0}^{x} Q_{n}^{\prime}(s) d s, \quad \text { and } \quad P_{n}(x):=\int_{0}^{x} P_{n}^{\prime}(s) d s .
$$

Then for $x \in I$ we have

$$
\left|P_{n}(x)\right| \vee\left|P_{n}^{\prime}(x)\right| \vee\left|Q_{n}(x)\right| \vee\left|Q_{n}^{\prime}(x)\right| \leq 2\left(\|u\|_{\infty}+1\right),
$$

and, as $n \rightarrow \infty$,

$$
\begin{gathered}
P_{n}(x) \rightarrow x^{+} \quad P_{n}^{\prime}(x) \rightarrow\left\{\begin{array}{ll}
0, & x<0 ; \\
1, & x \geq 0 .
\end{array} \quad P_{n}(x) / x \rightarrow \begin{cases}0, & x \leq 0 ; \\
1, & x>0 .\end{cases} \right. \\
Q_{n}(x) \rightarrow x^{-} \quad Q_{n}^{\prime}(x) \rightarrow\left\{\begin{array}{ll}
1, & x<0 ; \\
0, & x \geq 0 .
\end{array} \quad Q_{n}(x) / x \rightarrow \begin{cases}1, & x<0 ; \\
0, & x \geq 0 .\end{cases} \right.
\end{gathered}
$$

It follows by (2.11) and (2.13) that the sequences $\left(P_{n}(u)\right)_{n \in \mathbb{N}},\left(Q_{n}(u)\right)_{n \in \mathbb{N}}$, and also $\left(P_{n}(u) Q_{n}(u)\right)_{n \in \mathbb{N}}$ are bounded in $D(\mathcal{E})$. As well, we have $P_{n}(u) \rightarrow u^{+}, Q_{n}(u) \rightarrow u^{-}$, and $P_{n}(u) Q_{n}(u) \rightarrow 0$ in $L^{2}(E ; m)$, so by using the Lemma from the appendix we can find a common subsequence $\left(n_{i}\right)_{i \in \mathbb{N}}$ so that

$$
\begin{gathered}
(1 / N) \sum_{i=1}^{N} P_{n_{i}}(u) \rightarrow u^{+}, \\
(1 / N) \sum_{i=1}^{N} Q_{n_{i}}(u) \rightarrow u^{-}, \\
\text {and } \quad(1 / N) \sum_{i=1}^{N} P_{n_{i}}(u) Q_{n_{i}}(u) \rightarrow 0,
\end{gathered}
$$

each of these sequences converging strongly in $D(\mathcal{E})$. 
We now show that $\Gamma_{1}\left(u^{+}, u^{-}\right)=0$. From condition(c), for any $w \in D$ we have

$$
\Gamma_{1}\left(P_{n_{i}}(u) Q_{n_{i}}(u), w\right)=P_{n_{i}}^{\prime}(u) \Gamma_{1}\left(Q_{n_{i}}(u), w\right)+Q_{n_{i}}^{\prime}(u) \Gamma_{1}\left(P_{n_{i}}(u), w\right),
$$

and hence

$$
\frac{1}{N} \sum_{i=1}^{N} \Gamma_{1}\left(P_{n_{i}}(u) Q_{n_{i}}(u), w\right)=\frac{1}{N} \sum_{i=1}^{N} P_{n_{i}}^{\prime}(u) \Gamma_{1}\left(Q_{n_{i}}(u), w\right)+\frac{1}{N} \sum_{i=1}^{N} Q_{n_{i}}^{\prime}(u) \Gamma_{1}\left(P_{n_{i}}(u), w\right) .
$$

By (2.16) we have $L^{1}$-convergence so we can find a common subsequence $\left(N_{k}\right)_{k \in \mathbb{N}}$ that, $m$-a.e. on $E$, satisfies

$$
\begin{aligned}
& \left(1 / N_{k}\right) \sum_{i=1}^{N_{k}} \Gamma_{1}\left(P_{n_{i}}(u), w\right) \rightarrow \Gamma_{1}\left(u^{+}, w\right), \\
& \left(1 / N_{k}\right) \sum_{i=1}^{N_{k}} \Gamma_{1}\left(Q_{n_{i}}(u), w\right) \rightarrow \Gamma_{1}\left(u^{-}, w\right), \\
& \text { and } \quad\left(1 / N_{k}\right) \sum_{i=1}^{N_{k}} \Gamma_{1}\left(P_{n_{i}}(u) Q_{n_{i}}(u), w\right) \rightarrow 0 .
\end{aligned}
$$

We note that

$$
\left|\Gamma_{1}\left(Q_{n}(u), w\right)\right| \leq \sup _{x \in I}\left|Q_{n}^{\prime}(x)\right|\left|\Gamma_{1}(u, w)\right| \leq 2\left|\Gamma_{1}(u, w)\right|
$$

and

$$
\left|\Gamma_{1}\left(P_{n}(u), w\right)\right| \leq \sup _{x \in I}\left|P_{n}^{\prime}(x)\right|\left|\Gamma_{1}(u, w)\right| \leq 2\left|\Gamma_{1}(u, w)\right|
$$

so that the sequences $\left(\Gamma_{1}\left(Q_{n}(u), w\right)\right)_{n \in \mathbb{N}}$ and $\left(\Gamma_{1}\left(P_{n}(u), w\right)\right)_{n \in \mathbb{N}}$ are bounded $m$-almost everywhere. We use this boundedness, along with $(2.19)$ and the fact that $P_{n}^{\prime}(u) \rightarrow 1_{(u \geq 0)}$ and $Q_{n}^{\prime}(u) \rightarrow 1_{(u<0)} m$-a.e., and take pointwise limits along the subsequence $\left(N_{k}\right)_{k \in \mathbb{N}}$ in (2.18) to obtain

$$
0=1_{(u \geq 0)} \Gamma_{1}\left(u^{-}, w\right)+1_{(u<0)} \Gamma_{1}\left(u^{+}, w\right)
$$

In other words, $1_{(u \geq 0)} \Gamma_{1}\left(u^{-}, w\right)=0$, and $1_{(u<0)} \Gamma_{1}\left(u^{+}, w\right)=0$ for all $w \in D$. By continuity these equations extend to all $w \in D(\mathcal{E})$. In particular, $\Gamma_{1}\left(u^{+}, u^{+}\right)=0$ on $(u<0)$ and $\Gamma_{1}\left(u^{-}, u^{-}\right)=0$ on $(u \geq 0)$. This implies that

$$
\left|\Gamma_{1}\left(u^{+}, u^{-}\right)\right| \leq K \Gamma_{1}\left(u^{+}, u^{+}\right)^{1 / 2} \Gamma_{1}\left(u^{-}, u^{-}\right)^{1 / 2}=0
$$

on all of $E$. 
Now for $n, m \in \mathbb{N}$ we have

$$
\begin{aligned}
\mathcal{E}\left(P_{n}(u), Q_{m}(u)\right) & =\int \Gamma_{1}\left(P_{n}(u), Q_{m}(u)\right)+P_{n}^{\prime}(u) \Gamma_{2}(u, u)\left(Q_{m}(u) / u\right) d m \\
& +\int\left(P_{n}(u) / u\right) \Gamma_{3}(u, u) Q_{m}^{\prime}(u)+\left(P_{n}(u) / u\right) \Gamma_{4}(u, u)\left(Q_{m}(u) / u\right) d m .
\end{aligned}
$$

Using dominated convergence and letting first $n \rightarrow \infty$ and then $m \rightarrow \infty$ we get

$$
\begin{aligned}
\mathcal{E}\left(u^{+}, u^{-}\right) & =\int \Gamma_{1}\left(u^{+}, u^{-}\right)+1_{(u \geq 0)} \Gamma_{2}\left(u^{+}, u^{-}\right) 1_{(u<0)} d m \\
& +\int 1_{(u>0)} \Gamma_{3}\left(u^{+}, u^{-}\right) 1_{(u<0)}+1_{(u>0)} \Gamma_{4}\left(u^{+}, u^{-}\right) 1_{(u<0)} d m \\
& =0 .
\end{aligned}
$$

An application of Lemma 2.2 completes the proof.

Comment. If $\Gamma_{1}$ satisfies the product rule in both entries, then conditions (a) and (b) can be dropped, and a much shorter proof is possible along the lines of equations (2.24) and (2.25).

\section{Appendix}

Lemma. Suppose $\left(u_{n}\right)_{n \in \mathbb{N}}$ is bounded in $D(\mathcal{E})$ and that $u_{n} \rightarrow u$ in $L^{2}(E ; m)$. Then $u_{n} \rightarrow u$ weakly in $D(\mathcal{E})$, and there exists a subsequence $\left(u_{n_{k}}\right)_{k \in \mathbb{N}}$ with the property that for any further subsequence $\left(u_{n_{k_{j}}}\right)_{j \in \mathbb{N}}$, the Cesaro means $(1 / N) \sum_{j=1}^{N} u_{n_{k_{j}}}$ converge strongly to $u$ in $D(\mathcal{E})$.

Proof. Without loss of generality we take $u=0$. The weak convergence of $u_{n}$ follows from [MR 92; Chapter I, Lemma 2.12]. We now select a subsequence with the second property. Take $n_{1}=1$, and if $n_{1}, n_{2}, \ldots, n_{N}$ have been chosen we select $n_{N+1}>n_{N}$ so that

$$
\sup _{k=1}^{N}\left|\mathcal{E}\left(u_{n_{k}}, u_{n_{N+1}}\right)\right| \leq 2^{-(N+1)}
$$

which is possible since $u_{n} \rightarrow 0$ weakly in $D(\mathcal{E})$. We note that for every $i \in \mathbb{N}$, we have

$$
\sum_{j>i}\left|\mathcal{E}\left(u_{n_{i}}, u_{n_{j}}\right)\right| \leq \sum_{N=1}^{\infty} 2^{-N}=1
$$


Letting $c:=\sup _{n} \mathcal{E}\left(u_{n}, u_{n}\right)$ we have for any further subsequence $\left(u_{n_{k_{j}}}\right)_{j \in \mathbb{N}}$,

$$
\begin{aligned}
\mathcal{E}((1 / N) & \left.\sum_{j=1}^{N} u_{n_{k_{j}}},(1 / N) \sum_{j=1}^{N} u_{n_{k_{j}}}\right) \\
& \leq\left(1 / N^{2}\right) \sum_{j=1}^{N} \mathcal{E}\left(u_{n_{k_{j}}}, u_{n_{k_{j}}}\right)+\left(2 / N^{2}\right) \sum_{i=1}^{N} \sum_{i<j \leq N} \mathcal{E}\left(u_{n_{k_{i}}}, u_{n_{k_{j}}}\right) \\
& \leq(c / N)+(2 / N),
\end{aligned}
$$

which converges to zero as $N \rightarrow \infty$.

\section{Acknowledgement.}

This work was completed during a visit to the Institut für Angewandte Mathematik at the University of Bonn. I would like to thank my host, Michael Röckner, for providing hospitality and a stimulating working environment. I would also like to acknowledge the financial support of the Sonderforschungsbereich 256 and the Natural Sciences and Engineering Research Council of Canada.

\section{References}

[An76] Ancona, A., Continuité des contractions dans les espaces de Dirichlet. In: Sem. Th. Potentiel. Paris No. 2. (ed. by F. Hirsch and G. Mokobodzki), Lecture Notes in Mathematics 563, 1-26. Springer-Verlag, New York-Berlin-Heidelberg 1976

[BH91] Bouleau, N., Hirsch, F., Dirichlet Forms and Analysis on Wiener Space. Walter de Gruyter, Berlin-New York 1991

[F80] Fukushima, M., Dirichlet Forms and Markov Processes. North Holland, Amsterdam 1980

[MOR93] Ma, Z.M., Overbeck, L., Röckner, M., Markov processes associated with semi-Dirichlet forms. Preprint 1993

[MR92] Ma, Z.M., Röckner, M., An Introduction to the Theory of (Non-Symmetric) Dirichlet Forms. Springer-Verlag, New York-Berlin-Heidelberg 1992

[MR93] -, Markov processes associated with positivity preserving coercive forms. Preprint 1993 